\title{
Broadband over Inverse Multiplexed xDSL Modems
}

\author{
Einar Edvardsen \\ Telenor R \& D \\ PO Box 83, 2027 Kjeller, Norway \\ Einar-paul.edvardsen@telenor.com
}

\begin{abstract}
The paper describes a new scenario for how to transform the existing telephone access network into a real broadband network. The objective is to introduce a low-cost network evolution scenario, which makes maximal use of the huge investments connected to this kind of networks. The existing network is sub-divided into a small cell network, each covering an area with an approximate radius of 1 kilometre. Inverse multiplexing of xDSL systems is used to aggregate bandwidths in the range of 100 to $1000 \mathrm{Mb} / \mathrm{s}$ between nodes in the network. VDSL modems able to carry about $25-50 \mathrm{Mb} / \mathrm{s}$, are used on the last section from the node to the customer premises. These modems provide all households within each geographical cell with real broadband access to a large common bandwidth.
\end{abstract}

Keywords: Inverse multiplexing, ATM, xDSL, twisted pairs, FSAN, ITUNET

\section{Background}

At the present time there is a common understanding that the vision of 'Fibre-ToThe-Home' (FTTH) still belongs to an undefined future. This is both due to the fact that the demand for broadband services has not matured yet, that the related investments is supposed to be enormous and that such a huge civil work will take long time to accomplish. Other intermediate solutions are therefore looked upon to be more realistic.

FTTC (Fibre-To-The-Cabinet) is one of the approaches that have been adopted by the FSAN (Full Service Access Network) group. The idea behind FTTC is to utilise the existing telephone access network from the cabinet to the customer, but use optical fibre cables on sections between the cabinets and the broadband switch. By doing it this way, optical fibre cables will only be installed on sections were the costs can be shared by large numbers of customers, while the existing infrastructure will be used on the rest. In practice this means that ADSL or VDSL technology is supposed to play a major role in the access network in order to make available the necessary bandwidth on the last mile to the customers.

Though the FTTC is flexible and less expensive than the FTTH scenario, it still involves huge investments. In areas were cable ducts do not exist, optical cables will have to be buried in the ground. Even in cases were such ducts do exist, the cost of fibre cables and related installation work will reach considerable amounts and it will take time to accomplish - time that telecom operator companies possibly do not have. 
New competitors entering the market are offering broadband access over power-lines, radio, satellites and CATV networks. To maintain their market shares, the owners of the telephone cable network will have to meet this challenge by upgrading their networks. As we shall see, their potential performance is remarkable, and their possibility to still keep ahead in the race for broadband provisioning is rather good.

\subsection{The Capacity of Telephone Cables}

Though each single twisted pair in a telephone cable has a limited bandwidth, the total capacity of a telephone cable can nevertheless be very large. This is due to the fact that telephone cables contain large numbers of copper pairs, each of them can by utilising xDSL technology, provide a relative large bandwidth over a certain distance. If the capacity of each single pair could be summed, the aggregated capacity would be huge - see figure 1 below. Inverse multiplexing is the technology that enables such aggregation of capacity from a number of lower bandwidth digital channels.

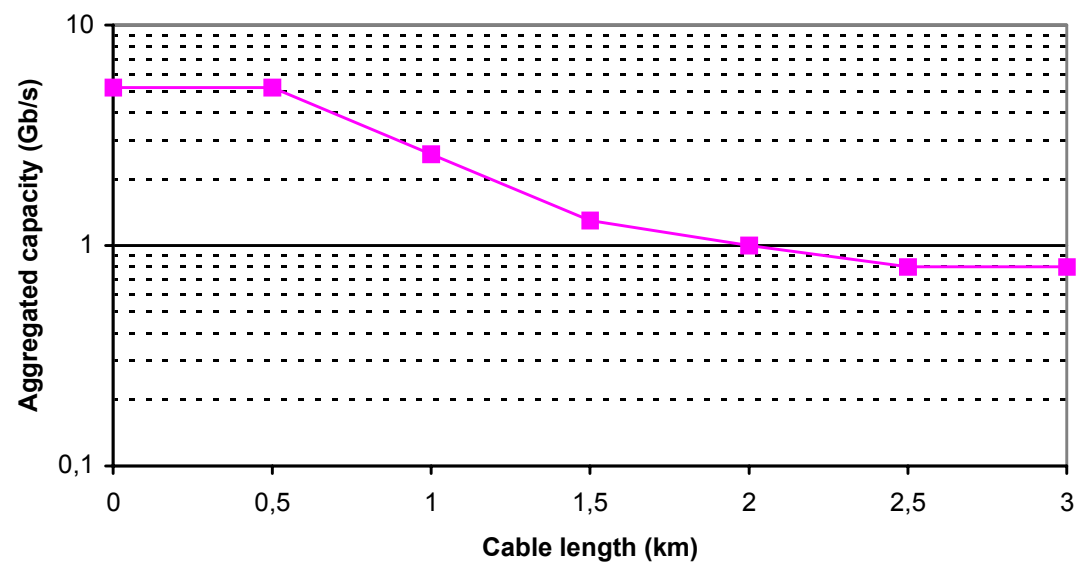

Fig. 1. Capacity of one hundred twisted pairs as a function of the distance

The figure shows the aggregated bandwidth of a telephone cable with 100 twisted pairs as a function of line length by using standard xDSL modems. A telephone cable with one hundred twisted pairs are in this context a small cable. The graphs indicate also that within reasonable cable lengths, the capacity of one hundred twisted pairs is in the range of several gigabits, i.e. a capacity that today is far beyond what is needed in residential access networks.

These brief calculations are of course not exact. Noise and cross talk influence on the obtainable bandwidth, resulting in less performance. Nevertheless they do indicate 
that the telephone cables are much more powerful than we are used to believe. The question is whether the bandwidth of this network can be exploited in an efficient way, thus opening a new possibility of how to provide real broadband access to the general public.

\section{Technology}

\subsection{Inverse Multiplexing}

Inverse multiplexing is used to aggregate bandwidth from a number of 'lines with smaller bandwidths' as shown in figure 2 below:

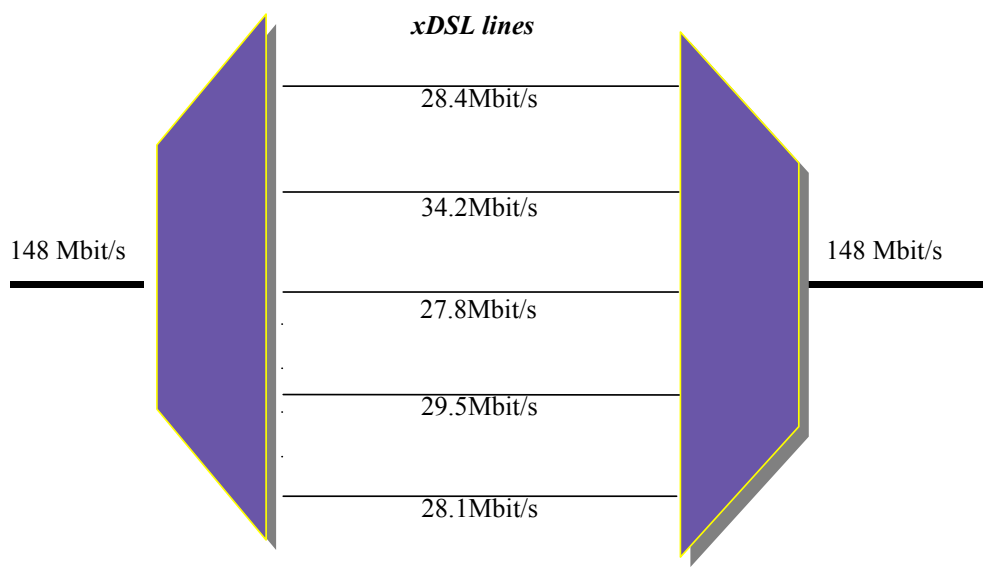

Fig. 2. The principle of inverse multiplexing

The incoming traffic stream from the left is distributed sequentially over a number of lower bitrate lines, and reassembled at the other end.

Inverse multiplexing is standardised by a number of standardisation organisations, such as ITU, ETSI and ATM Forum. ATM Forum's AF-PHY-0086.001 covers inverse multiplexing E1/DS1 links to aggregate bandwidths up to E3/DS3 rates. Though it from a technical point of view is possible to multiplex channels running various bitrates, this has so far not been covered by any official standard yet. Since 
xDSL modems provide bandwidths deviating from the standardised hierarchy, the standards cannot be applied unless it is used on circuit emulated E1/DS1 channels.

It exists several methods of how to perform inverse multiplexing of packet based traffic (ATM). One of them is shown in figure 3, where the principle of sequence numbering is used.

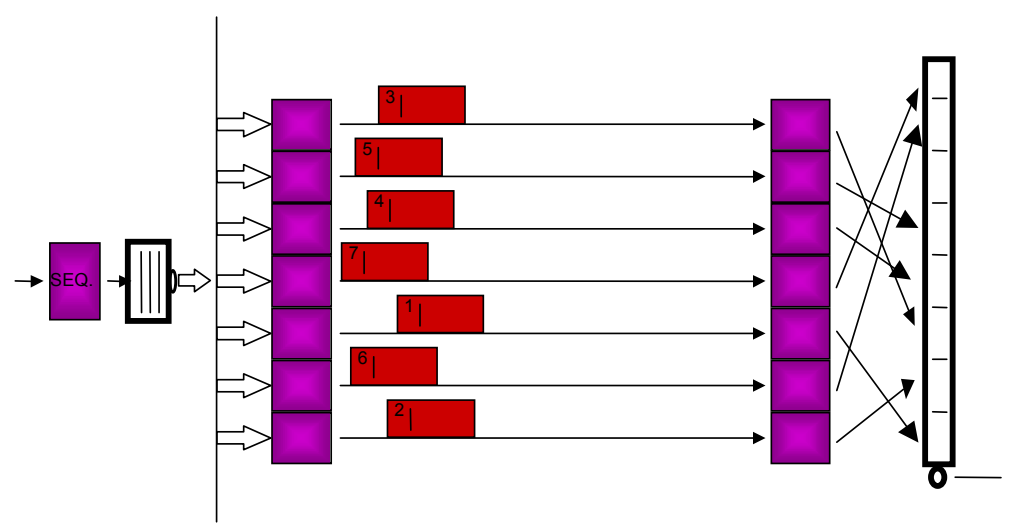

Fig. 3. Inverse multiplexing by use of sequence numbering over xDSL lines

Each cell arriving from the left is given a sequence number before transmitted over one of the available lines. Though not indicated on this figure, the bandwidth of each line may differ from each other. One line can perform $13 \mathrm{Mb} / \mathrm{s}$, while an other may perform 19,3 Mb/s. The cells will therefore arrive out of order at the receiver end, and has to be intermediately stored in the receiver buffer on right side in order to reestablish the sequential order. Since it is mandatory that inverse multiplexers should be transparent for traffic streams, and that the ATM cell itself does not contain any field useful for carrying the sequence number, it must be transferred as a tag to the cell. The cell length will therefore deviate from the ATM standard (53 bytes). However, the interface between the two terminals of the inverse multiplexer can be looked upon as an internal interface, and a deviation from the standards is therefore acceptable.

Mixing of lines with low speed and different bandwidths may create delay and cell delay variation (CDV) that are unacceptable for certain types of traffic. To meet requirements from the various traffic types, each of them may have to use dedicated line groups with properties that match their demands.

\subsection{The network concept}

The proposed network concept, from here of named the IMUX concept, is based upon using the existing telephone access network infrastructure. In practice it means that the existing telephone cables are used to provide broadband access to the public. In the general structure of the new network, new access nodes have to be installed near 
the existing street cabinets. These network nodes must perform both inverse multiplexing to establish the transmission links to other nodes, and statistical multiplexing to concentrate the traffic from the users. Figure 4 below shows a functional description of such a network node.

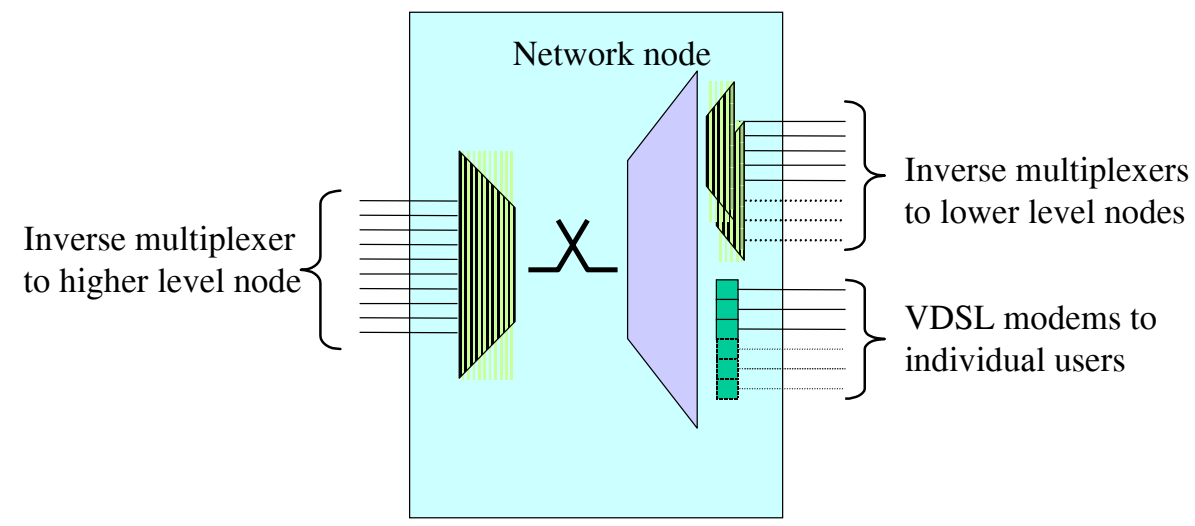

Fig. 4. The network Node

Figure 5 below shows the principal structure of the new network. The nodes in the network are installed near the distribution cabinets in the old telephone network in order to have easy access to the necessary copper pairs. The nominal distance between the nodes is about 2 kilometres. Each node covers an area with an approximate radius of 1 kilometre, which makes it possible to provide $25-50 \mathrm{Mb} / \mathrm{s}$ to each of the customers.

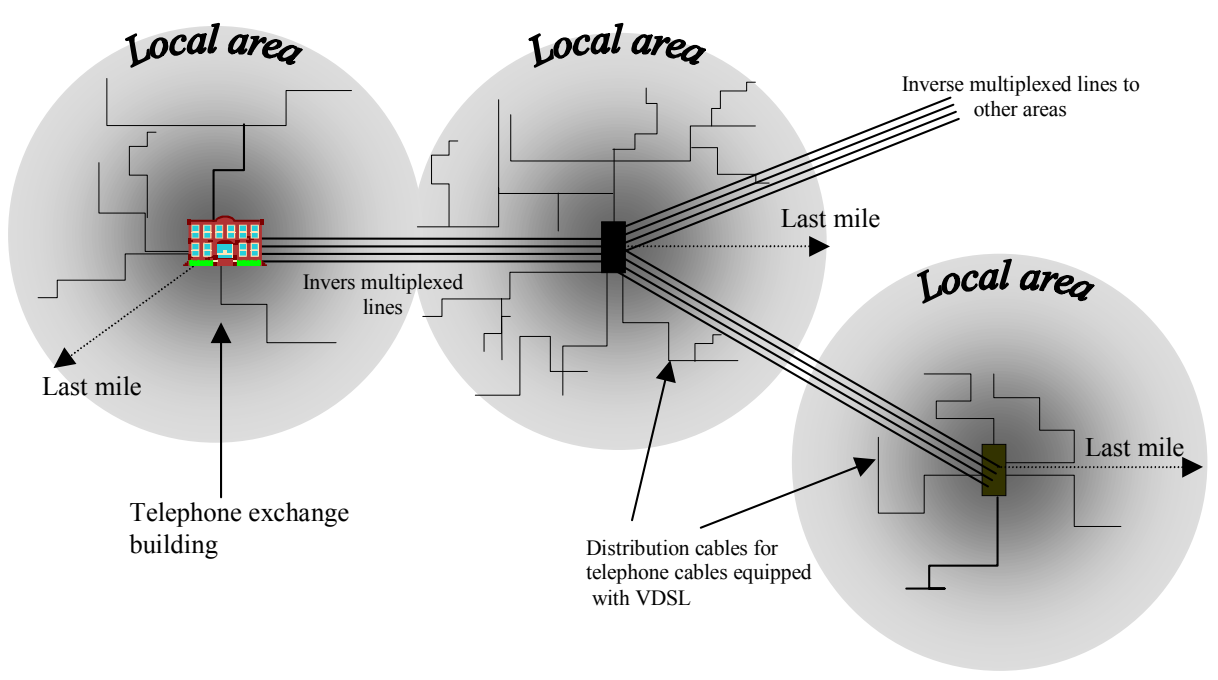

Fig. 5. The architecture of the IMUX network 
Inverse multiplexing over a number of copper pairs running VDSL modems is used to aggregate the requested bandwidth between the main ATM switch located at the telephone exchange building, and nodes in the network. The link capacity needed between the nodes is estimated to be in the range of 100 to $1000 \mathrm{Mb} / \mathrm{s}$, which can be obtained by inverse multiplexing of a few tens of lines.

Each network node performs both inverse multiplexing and statistical multiplexing of traffic flows from the individual users. The users are connected to the nearest node by VDSL modems, giving them access to a large, common bandwidth shared among them. The nodes will be equipped with standard ATM signalling enabling use of SVCs (Switched Virtual Connections) with QoS (Quality of Service) as defined by relevant standards.

The structure of this network is similar to the recommendation from the FSAN (Full Service Access Network) consortium. The difference is that FSAN recommends optical fibres between the nodes, while this approach is based upon copper. However, due to the similarities between the two concepts, it is easy to adopt the technology that fits the need best in each case. On sections were optical cables can be installed at low cost, fibre cables are the natural choice. But on sections where optical cables cannot be installed at a reasonable cost, inverse multiplexing is the choice. The two approaches go hand in hand - they will complement each other in a perfect way that both the operators and the public may profit on.

\section{The ACTS Project AC309 ITUNET}

To promote the network concept and to pave the way for commercial products needed to implement this kind of network, the EU financed ACTS project AC309 ITUNET was initiated. The project started in the middle of March 1998 and was terminated at the end of March 2000.

The main objectives of the project was to study how the existing access network infrastructures can be upgraded using xDSL technologies to form a cost-efficient integrated service network providing the necessary capacity and functionality for broadband services.

In order to identify eventual limitations of the technology and the concept, an experimental access node was developed. The node performed inverse multiplexing of up to 16 VDSL lines and statistical multiplexing of up to 28 user modems.

Secondly, since the existing infrastructure imposes strong requirements to the location of network nodes, it was of special interest to carry through implementability studies from real networks in order to see if the network could be transformed according to the concept. Were there copper pairs enough on all sections? Were the line lengths within acceptable ranges? How about power supply?

And thirdly, it was necessary to see if it was possible to realise the necessary bandwidth. Video services like TV broadcasting (multi-casting) and Video-onDemand were chosen to be the killer applications that every household wanted to subscribe to.

The project also carried through in-depth calculations and evaluation of the cost related to establishing access networks based on inverse multiplexing.

The project counted telecom operators and industrial partners from France, Switzerland and Norway. 


\section{Evaluation}

In order to have a rough evaluation of the implementability of such a network and also some imagination about the cost to establish it, several case studies were performed. The case studies covered various areas in Norway and Switzerland. Rural areas as well as urban areas were investigated.

In the following sub-sections one of these case studies is described in details, while only summary information about the others is given.

\subsection{The Upgraded Network}

NorVillageA is a small village in a rural area with population consisting mainly of farmers and private households. The village had 728 telephone subscribers in total. 384 of them lived closer to the telephone exchange building than 1000 metres, thus one could provide $25-50 \mathrm{Mb} / \mathrm{s}$ to each of them directly from the exchange building with single user VDSL modems. The remaining subscribers, 344, were connected over remote access nodes. See figure 6 below.

To connect all customers, 18 access nodes had to be installed. The average number of customers connected to each access node was 19. Due to the topology and the existing cable infrastructure access nodes had to be installed even at locations with very few customers $(<5)$. As a result of this, the number of network nodes became higher than expected.

The case study also uncovered that due to the above non-optimal location of the network nodes, up to 4 cascaded nodes were needed to reach the most distant users.

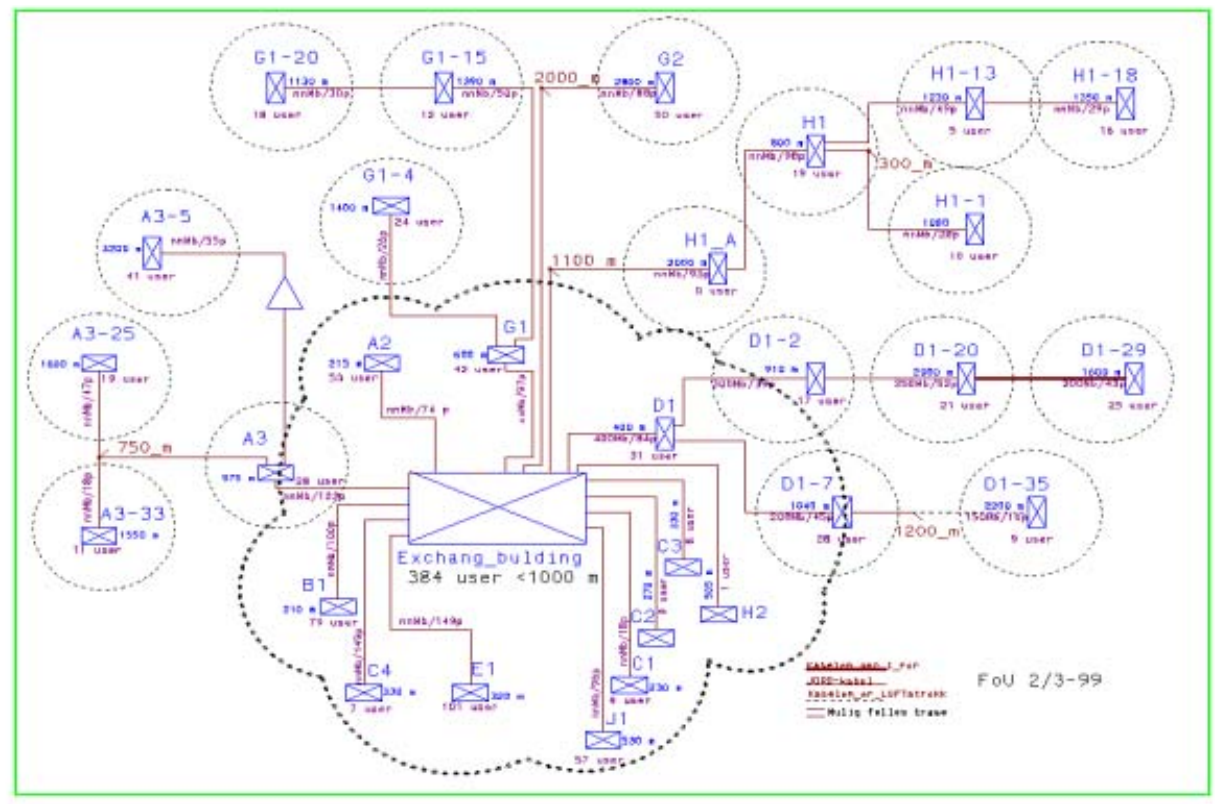

Fig. 6. The upgraded network 


\subsection{Cost Comparison}

Since the FTTC and the IMUX architectures only differ as regards one item, optical fibres vs. inverse multiplexers on the feeder link, the cost comparison could be made very simple. The number of VDSL modems needed in the two concepts is almost equal since both concepts recommend use of modems between the access node and the customers. The number of end-user modems is much higher than the number of modems used for establishing link capacities. It is assumed that IMUX concept needs $10-15 \%$ more modems than the FTTC concept. Consequently, it was not considered necessary to compare other costs than the investment related to

- Optical fibres, optical termination and civil work for the FTTC concept

- Vs. inverse multiplexers and modems for the IMUX concept

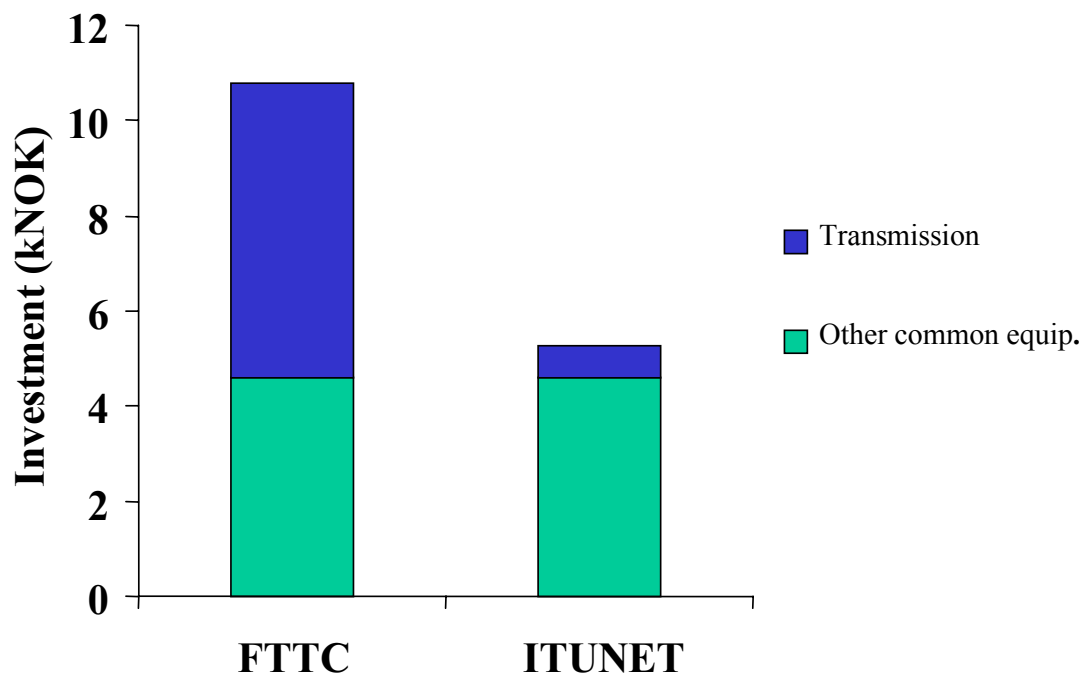

Fig. 7. Comparison of investment cost

The result from the cost comparison (Figure 7) shows that the investment to establish link capacities between the nodes in the network can be reduced by approximately $90 \%$ by utilising IMUX technology. This percentage has been verified by a number of case studies in different countries as well as different topologies.

\section{Recommendations}

The IMUX concept is introduced as an independent strategy for how to build a broadband access network able to provide both todays and tomorrow's services to the general public. This does not mean that other solutions should be excluded. The concept is well adapted to other approaches, such as the above-mentioned FTTC approach. These two strategies are complementary in the sense that one easily can combine sub-nets based upon the two technologies without large influence on the overall network structure. Both of the two approaches rely on utilisation of the 
existing telephone network. Optical fibres can supplement a network based upon the IMUX idea, as well as inverse multiplexers can supplement a network based upon in the FTTC concept.

The IMUX concept is an inexpensive and fast method to establish a broadband access network. The investment cost to establish link capacities can be reduced by 90 $\%$ compared to the FTTC concept and the network can be realised very fast since civil work is reduced to a minimum. The will be no need to acquire ground and getting permissions to dig cable trenches.

The IMUX concept only requests a moderate increase of the total number of modems in the network. The estimated increase is approximately $10-15 \%$, which is judged to be insignificant as regards maintenance cost.

An optical cable has more capacity than a telephone cable, but the bandwidth of the latter one will in most cases be sufficient. Bandwidths in the range of $\mathrm{Gb} / \mathrm{s}$ can relatively easily be achieved.

None of the concepts need to influence on the telephony service. Both analogue and digital telephony can be transmitted in a separate frequency band on the twisted pairs.

The complexity of the remote network nodes in the two cases will only be slightly increased since inverse multiplexers will have to be integrated into the IMUX equipment. 